\title{
Ethanol extract of Sanguisorba officinalis L. inhibits biofilm formation of methicillin-resistant Staphylococcus aureus in an ica-dependent manner
}

\author{
Xiaolin Chen, ${ }^{*}$ Fei Shang, ${ }^{*}$ Yajing Meng, ${ }^{*}$ Long Li, ${ }^{*}$ Yunmei Cui, $†$ Ming Zhang, ${ }^{*}$ Kezong Qi, $\ddagger^{1}$ and Ting Xue ${ }^{* 1}$ \\ *School of Life Sciences, \\ †School of Sciences, and \\ ¥College of Animal Science and Technology, Anhui Agricultural University, Hefei, Anhui 230036, China
}

\begin{abstract}
Methicillin-resistant Staphylococcus aureus (MRSA) is an important nosocomial pathogen that shows resistance to many antibiotics and is usually associated with serious infections. Having the ability for biofilm formation increases resistance to antibiotics. Sanguisorba officinalis $\mathrm{L}$. is a perennial plant that is distributed in the northern districts of China and has been used as a traditional Chinese medicine. In this study, the effect of S. officinalis on MRSA strain SA3 isolated from a dairy cow with mastitis was evaluated by testing the growth and biofilm formation ability of MRSA cultured with or without ethanol extracts of $S$. officinalis. The results showed that the ethanol extract of $S$. officinalis strongly inhibited the biofilm formation of MRSA. With a confocal laser scanning microscope system, we observed that the biofilm structure of the test group with the addition of $S$. officinalis appeared looser and had less biomass compared with the control group without $S$. officinalis. Furthermore, we found that the transcript levels of the $i c a A D B C$ operon remarkably decreased upon addition of the ethanol extract of $S$. officinalis, indicating that S. officinalis inhibits biofilm formation of MRSA in an ica-dependent manner.
\end{abstract}

Key words: Sanguisorba officinalis L., methicillinresistant Staphylococcus aureus, biofilm

\section{INTRODUCTION}

Staphylococcus aureus is an important pathogen that is a common cause of community- and hospital-acquired infections. Staphylococcus aureus can be well adapted to the human host by adhering to specific host substrates, evading host defenses, and resisting antibiotic therapy (Greenberg et al., 1989; Patti et al., 1994; Park et al., 1996; Joh et al., 1999; Hussain et al., 2002; Fedtke et al., 2004; Shanks et al., 2005). A variety of virulence factors

Received June 2, 2015.

Accepted August 22, 2015.

${ }^{1}$ Corresponding authors: qkz@ahau.edu.cn and xuet@ahau.edu.cn and specific mechanisms contribute to their ability to develop chronic infections in the host. One important way in which these bacteria adapted is through biofilm formation (Stewart, 2002; Gowrishankar et al., 2012). Biofilms are a population of bacteria growing on biotic and abiotic surfaces that embed themselves in a selfproduced extracellular matrix of exopolysaccharide, proteins, and some micromolecules, such as DNA (Gowrishankar et al., 2012). The structure of biofilm constitutes a protected environment for the growth of bacteria by restricting antibiotic access and shielding the bacterial pathogen from host immune defenses (Joh et al., 1999; Nguyen et al., 2008; Atshan et al., 2012).

Methicillin-resistant Staphylococcus aureus (MRSA) is a bacterium that has developed resistance, through the process of natural selection, to $\beta$-lactam antibiotics, which include penicillins (e.g., methicillin, dicloxacillin, nafcillin, oxacillin) and cephalosporins (Barrett et al., 1968; Ubukata et al., 1985; Matsuhashi et al., 1986). Serious infections are associated with MRSA, and it poses a serious threat to public health worldwide. The ability of biofilm formation in MRSA can lead to resistance to most currently used antibiotics (Ando et al., 2004). Therefore, biofilm-forming MRSA infections are life-threatening. In recent years, with the extensive use of implanted medical devices for diagnostic and therapeutic purposes, which can be easily colonized by biofilm-forming staphylococci (O'Gara, 2007), the early identification and prevention of the most serious nosocomial infections caused by biofilm-forming MRSA become more urgent and important for medical care.

Sanguisorba officinalis L. is a perennial plant that is distributed in the northern districts of China. The dried roots of $S$. officinalis display hemostatic, antiallergic, analgesic, and astringent properties, and have been used in traditional Chinese medicine for the treatment of burns, scalds, and internal hemorrhage (Liu et al., 2005; Cai et al., 2012). Several modern pharmacological studies have shown that the root contains various saponin components, which have been proven to be the main anticancer agents in some natural plants. Recently, some authors have demonstrated that aqueous extracts 
of S. officinalis had obvious anticancer effects by inducing cancer cell apoptosis and inhibiting angiogenesis, which has the possibility of being developed as a breast cancer therapeutic agent (Bastow et al., 1993; Liu et al., 2005). In addition, several other investigations revealed that terpenoids and triterpenoid glycosides from this herb have significant antioxidant and neuroprotective activities (Yokozawa et al., 2000, 2002; Liu et al., 2005; Nguyen et al., 2008; Zhang et al., 2012).

In the current study, we investigated the effect of $S$. officinalis on MRSA. The growth and biofilm formation of MRSA cultured with or without ethanol extracts of S. officinalis was tested by performing colony-forming unit assays and microplate assays, as well as using a confocal laser scanning microscope (CLSM) system. Results showed that the ethanol extract of $S$. officinalis has a significant inhibitory effect on biofilm formation in MRSA. In addition, the mechanism of how $S$. officinalis inhibits biofilm formation was investigated by using real-time reverse transcription (RT) PCR. The results indicated that $S$. officinalis inhibits the biofilm formation of MRSA in an ica-dependent manner.

\section{MATERIALS AND METHODS}

\section{Bacterial Strain and Growth Condition}

Staphylococcus aureus MRSA strain SA3 used in our study were isolated from a dairy cow with mastitis. Strain SA3 is a mecA-positive multidrug-resistant strain. The antibiotic sensitivity assays showed that strain SA3 is sensitive to chloromycetin and vancomycin, and resistant to ampicillin, erythromycin, and oxacillin. Strains were grown at $37^{\circ} \mathrm{C}$ in tryptic soy broth (TSB) containing $0.25 \%$ glucose medium (Oxoid, Basingstoke, UK).

\section{Ethanol Extraction of S. officinalis}

The ethanol extract of $S$. officinalis was prepared followed with the method described previously (Yu et al., 2011). Sanguisorbae radix, the root of $S$. officinalis, was dried at $50^{\circ} \mathrm{C}$ and crushed into a powder $(0.3-0.5 \mathrm{~cm} \times$ $0.1-0.3 \mathrm{~cm})$ with a blender, and the crude powder was extracted with ethanol at $70^{\circ} \mathrm{C}$ for $3 \mathrm{~h}$. Subsequently, the ethanol extracts were evaporated at $60^{\circ} \mathrm{C}$ under pressure, the powder was dissolved in $95 \%$ ethanol to make a stock solution $(50 \mathrm{mg} / \mathrm{mL})$, and the solution was filtered by a syringe filter with $0.22-\mu \mathrm{m}$ pores.

\section{Antimicrobial Activity Assay}

Colonies of MRSA strain SA3 were placed into $2 \mathrm{~mL}$ of TSB medium and cultivated at $37^{\circ} \mathrm{C}$ with shaking at $200 \mathrm{rpm}$ for $16 \mathrm{~h}$. Then, the overnight cultures were inoculated into fresh TSB medium and diluted to a final concentration of 0.05 by optical density at 600 $\mathrm{nm}$ before being dispensed into 96-well plates (Costar, Corning, Steuben, NY) containing serial dilutions of ethanol extracts of $S$. officinalis Plates were incubated at $37^{\circ} \mathrm{C}$ for $12 \mathrm{~h}$ and then 10 -fold serial dilutions of cultures were performed by successive transfer $(0.1 \mathrm{~mL})$ through 7 microfuge tubes containing $0.9 \mathrm{~mL}$ of TSB. Next, $100-\mu \mathrm{L}$ dilutions were dropped on Luria-Bertani agar plates and viable colonies were counted via their colony-forming units on TSB agar plates after incubated at $37^{\circ} \mathrm{C}$ for $24 \mathrm{~h}$. The survival rate of the control group without exposure to S. officinalis was designated as $100 \%$. Colony-forming units of the test groups were all compared with that of the control group. Experiments were repeated 3 times with 4 parallels.

\section{Biofilm Assays}

The method for biofilm quantification was performed as described previously and modified as described herein (Mack et al., 2000; Sadovskaya et al., 2005; Yu et al., 2012). We grew MRSA strain SA3 in TSB for 16 $\mathrm{h}$ and diluted it 1:100 into fresh TSB. The diluted cultures were transferred into sterile 96-well flat-bottomed tissue culture plates and incubated at $37^{\circ} \mathrm{C}$ for $24 \mathrm{~h}$. Ethanol extracts of $S$. officinalis were added to the TSB medium with diluted cultures at different concentrations. The adherent bacteria were stained with crystal violet, and the excess stain was washed off gently with slowly running water. The biomass of the biofilm was determined using a MicroELISA auto-reader (BioRad, Hercules, CA) at a wavelength of $560 \mathrm{~nm}$ under single-wavelength mode (Ziebuhr et al., 1997; Pozzi et al., 2012). Laser scanning confocal microscopy was performed on a Zeiss LSM710 system (Carl Zeiss GmbH, Jena, Germany) with a $20 \times 0.8$ numerical aperture apochromatic objective. The strains were stained with acridine orange for fluorescence detection. The laser wavelength used for the CLSM was $488 \mathrm{~nm}$; Z-stacks were collected at $1-\mu \mathrm{m}$ intervals. Confocal parameters for wild-type biofilm detection were taken as standard settings. Each confocal experiment was repeated 4 times. The 3-dimensional biofilm images were rendered with Imaris 7.0 (Bitplane, Zurich, Switzerland).

\section{Total RNA Isolation, cDNA Generation, and Real-Time PCR Processing}

Overnight cultures of MRSA strain SA3 were diluted 1:100 in TSB containing $0.5 \%$ glucose and grown to the late exponential phase in 24-well plates (Costar). Cells were collected and resuspended in Tris-EDTA 
Table 1. Oligonucleotide primers used in this study

\begin{tabular}{ll}
\hline Primer name & Oligonucleotide $\left(5^{\prime}-3^{\prime}\right)$ \\
\hline rt-icaA-f & TTTCGGGTGTCTTCACTCTAT \\
rt-icaA-r & CGTAGTAATACTTCGTGTCCC \\
rt-icaB-f & CCTATCCTTATGGCTTGATGA \\
rt-icaB-r & CATTGGAGTTCGGAGTGA \\
rt-icaC-f & TACTGACAACCTTGAATTACCA \\
rt-icaC-r & AATAGCCATACCATTGACCTAA \\
rt-icaD-f & CCAGACAGAGGGAATACC \\
rt-icaD-r & AAGACACAAGATATAGCGATAAG \\
rt-icaR-f & TTATCTAATACGCCTGAGGAAT \\
rt-icaR-r & GGATGCTTTCAAATACCAACT \\
\hline
\end{tabular}

buffer ( $\mathrm{pH}$ 8.0) containing $10 \mathrm{~g} / \mathrm{L}$ of lysozyme and $40 \mathrm{mg} / \mathrm{L}$ of lysostaphin. After incubation at $37^{\circ} \mathrm{C}$ for 5 min, cells were prepared for total RNA extraction using the Trizol method (Invitrogen, Waltham, MA), and residual DNA was removed with DNase (RNase free; TaKaRa, Shiga, Japan). Real-time RT-PCR was performed with a PrimeScript 1st Strand cDNA synthesis kit and SYBR Premix Ex Taq (TaKaRa) using a StepOne real-time PCR system (Applied Biosystems, Thermo Fisher Scientific, Waltham, MA). The quantity of cDNA measured by real-time PCR was normalized to the abundance of $16 \mathrm{~S}$ cDNA (Chen et al., 2000). All of the real-time RT-PCR assays were repeated at least 3 times with similar results. The primers used in our study are listed in Table 1.

\section{Statistical Analysis}

The data were analyzed using the statistical software SPSS (ver. 19.0, IBM Corp., Armonk, NY) by a oneway ANVONA method; the test results were shown as mean $\pm \mathrm{SD}$. The paired $t$-test was used for statistical comparisons between groups. The level of statistical significance was set at a $P$-value of $\leq 0.01$.

\section{RESULTS}

\section{Inhibitory Effect of Ethanol Extract of S. officinalis on Staph. aureus}

To examine the antibacterial activity of the ethanol extract of S. officinalis against Staph. aureus in vitro, the antibacterial assays were performed. After exposure to the extract of $S$. officinalis at different concentrations for $12 \mathrm{~h}$ at $37^{\circ} \mathrm{C}$, the cells of MRSA strain SA3 were inoculated into fresh TSB and then spread onto the TSB agar plates. After cultivating for $24 \mathrm{~h}$ at $37^{\circ} \mathrm{C}$, the colony-forming units of the bacteria were counted and compared. As shown in Figure 1A, the survival rate of the control group without exposure to $S$. officinalis was designated as $100 \%$. With the increase
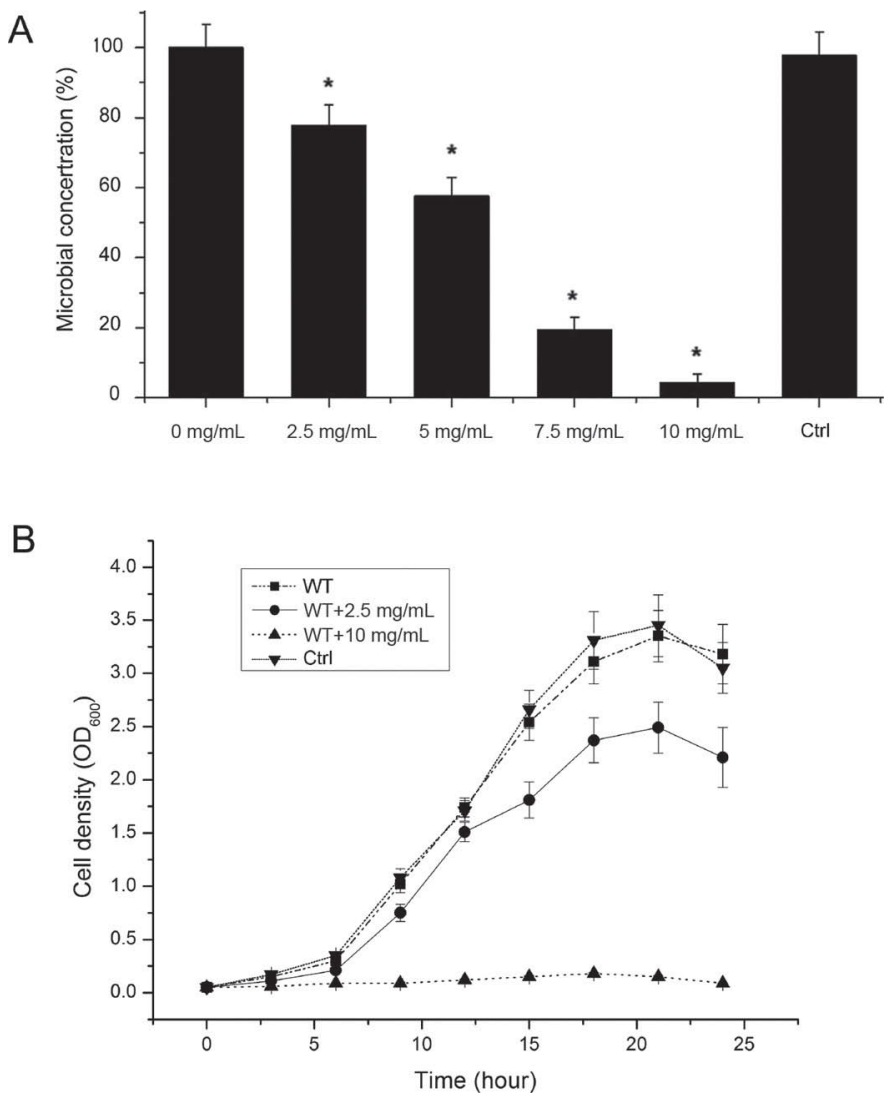

Figure 1. (A) Colony-forming unit assays of methicillin-resistant Staphylococcus aureus (MRSA) strain SA3. Colony counts of SA3 following $12 \mathrm{~h}$ of incubation at $37^{\circ} \mathrm{C}$ with or without ethanol extract of Sanguisorba officinalis were compared. The survival rate of the control group without exposure to S. officinalis was designated $100 \%$. The colony counts of the test group cultured with $S$. officinalis at different concentrations were all compared with that of the control group. An asterisk $(*)$ represents means significantly different from the control group with no ethanol extract of $S$. officinalis $(P<0.05)$. (B) The growth curves of MRSA strain SA3 cultured in tryptic soy broth medium with or without ethanol extract of $S$. officinalis. The results represent a mean of 3 independent experiments. $\mathrm{Ctrl}=$ a negative control in which the ethanol extract of $S$. officinalis was substituted by equal volume of ethanol.

in the concentration of $S$. officinalis L., the survival rate of MRSA strain SA3 decreased accordingly. When the concentration of $S$. officinalis reached $10 \mathrm{mg} / \mathrm{mL}$, the survival rate of cells dropped to $10 \%$. These data indicated that $S$. officinalis has antibacterial activity against Staph. aureus.

Furthermore, the growth rates of the cells were tested when they were grown in TSB medium with S. officinalis at different concentrations. The results showed that the growth rates of the bacteria decreased to a small extent when the external concentration of $S$. officinalis was $2.5 \mathrm{mg} / \mathrm{L}$ compared with the wild type without the addition of S. officinalis L.; when the concentration of S. officinalis was $10 \mathrm{mg} / \mathrm{mL}$, the growth of the bacteria 
was remarkably inhibited (Figure 1B). These data indicated that only at high concentrations can $S$. officinalis show considerable antibacterial activity against Staph. aureus.

\section{Inhibitory Effect of the Ethanol Extract of S. officinalis on Biofilm Formation of Staph. aureus}

To examine whether the ethanol extract of $S$. officinalis affects biofilm formation of Staph. aureus, we performed biofilm assays. The cells of MRSA strain SA3 were grown in TSB for $16 \mathrm{~h}$ and diluted 1:100 in fresh TSB. The ethanol extract of $S$. officinalis was added to the TSB medium to final concentrations of 0 , $1,2.5,5$, and $7.5 \mathrm{mg} / \mathrm{mL}$. Cultures were transferred to sterile 96 -well flat-bottomed tissue culture plates and incubated for $16 \mathrm{~h}$ at $37^{\circ} \mathrm{C}$. After crystal violet staining, we observed biofilm biomass in the 96 -well plates. As shown in Figure 2A, strains of the control group without ethanol extract of $S$. officinalis formed obvious biofilms, whereas with the increase of the concentration of $S$. officinalis biofilm formation of the bacteria significantly decreased, and when the concentration of $S$. officinalis reached $5 \mathrm{mg} / \mathrm{mL}$, no biofilm was observed. In addition, the quantity of biofilm formation was further tested using a MicroELISA autoreader (Thermo Scientific, Pittsburgh, PA). We found that biofilm quantity decreased with the increase of S. officinalis $L$ concentration (Figure 2A). To directly observe the effect of ethanol extract of $S$. officinalis on biofilm formation of Staph. aureus, we used the CLSM system and the strains were stained with acridine orange for fluorescence detection. After $12 \mathrm{~h}$ of incubation at $37^{\circ} \mathrm{C}$ in TSB containing $0.5 \%$ glucose, strains of the control group without ethanol extract of $S$. officinalis produced intact and rough biofilms, as monitored by CLSM (Figure $2 \mathrm{~B}$ ). In contrast, biofilms produced by strains of the experimental group with $2.5 \mathrm{mg} / \mathrm{mL}$ of ethanol extract of $S$. officinalis were nearly undetectable (Figure 2B). These data revealed an interesting phenomenon that although the antibacterial activity of S. officinalis at a low concentration is not obvious, the effect of that on biofilm formation is significant.

\section{Ethanol Extract of S. officinalis Significantly Inhibited MRSA Biofilm Formation by Decreasing Transcription of the icaADBC Operon}

To investigate the mechanism of how S. officinalis regulates biofilm formation in MRSA strain SA3, the transcript levels of biofilm-associated genes were tested by performing real-time RT-PCR experiments. We examined the transcript levels of the icaADBC operon, which encodes the polysaccharide intercellular adhesin
A

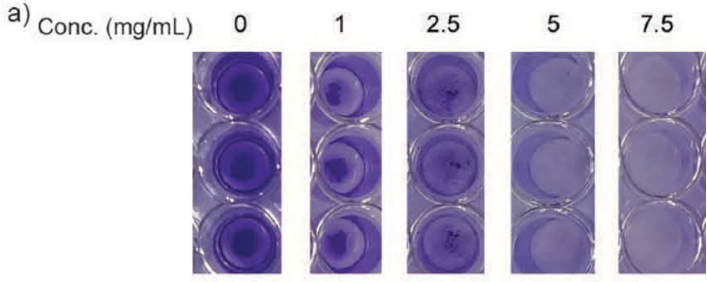

b)

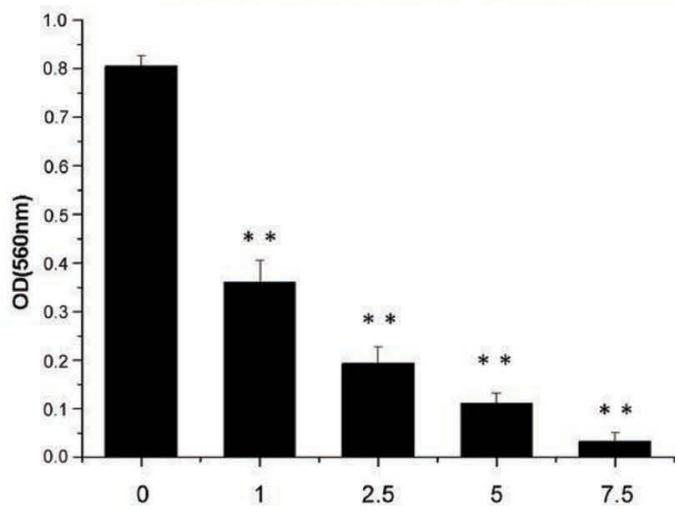

B

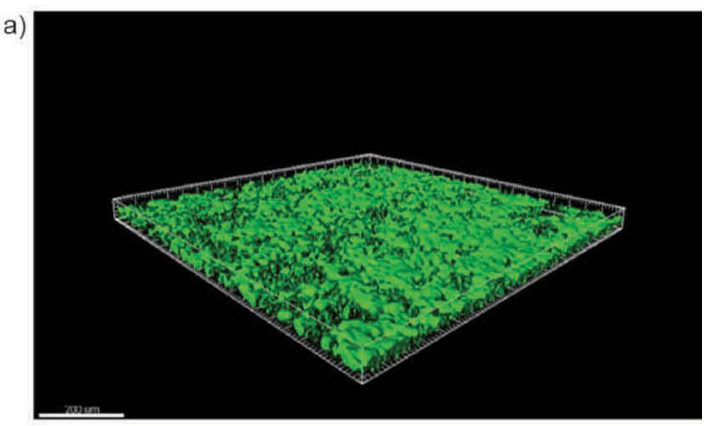

b)

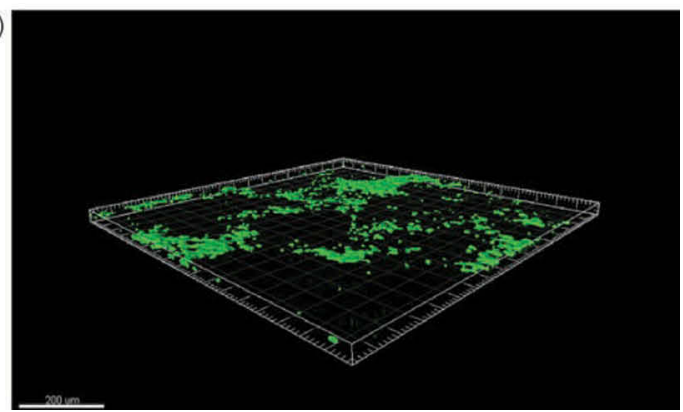

Figure 2. Effect of Sanguisorba officinalis on biofilm formation in methicillin-resistant Staphylococcus aureus (MRSA) strain SA3. Biofilm formation of SA3 cultured in tryptic soy broth containing $0.5 \%$ glucose in 96-well plates for $12 \mathrm{~h}$ at $37^{\circ} \mathrm{C}$ with or without ethanol extract of ethanol extract of $S$. officinalis (A, a) Photographs of the 96-well plates after staining with crystal violet; (b) cells that adhered to the plate after staining with crystal violet were measured by optical density at $560 \mathrm{~nm}$. (B) Biofilm integrity and fluorescence were monitored by CLSM: (a) control group with no ethanol extract of $S$. officinalis L., and (b) the external concentration of S. officinalis is 2.5 $\mathrm{mg} / \mathrm{mL}$. Biofilm integrity and green fluorescent protein (GFP) fluorescence were monitored by confocal laser scanning microscope and the 3-dimensional images were rendered with Imaris 7.0 (Bitplane, Zurich, Switzerland). The results represent a mean of 3 independent experiments. Double asterisks $\left({ }^{*}\right)$ means significantly different from the control group with no ethanol extract of $S$. officinalis $(P<0.01)$. 
(PIA) synthetases. The cells of MRSA strain SA3 were cultivated for $16 \mathrm{~h}$ in TSB with $S$. officinalis at concentrations of 0,1 , and $3 \mathrm{mg} / \mathrm{mL}$. Subsequently, the cells were harvested and the transcript levels of the ica operon were tested. As shown in Figure 3A, the transcript levels of $i c a A, i c a B, i c a C$, and $i c a D$ were significantly decreased upon the addition of $S$. officinalis L., and with the increase of $S$. officinalis concentration the inhibitory effect of $S$. officinalis on the ica operon was stronger, indicating that $S$. officinalis affects the transcription of the ica operon in a concentration-dependent manner. To further investigate how S. officinalis regulates the ica operon, we examined the transcript level of $i c a R$, which has been identified as the repressor of the $i c a$ operon. Results showed that the transcript level of $i c a R$ increased by adding $S$. officinalis in the culture medium in Staph. aureus. (Figure 3B), confirming that $S$. officinalis influences the ica operon through the transcriptional regulator $i c a R$.

\section{DISCUSSION}

Bacterial biofilms have become a major impediment to the cure of many chronic human infections, which are characterized by prolonged inflammation and respond poorly to conventional treatments (Secor et al., 2011). The resistance to antimicrobial agents among bacteria growing in biofilms is many times higher than that of their planktonic counterparts ( $\mathrm{Yu}$ et al., 2011). The insensitivity of biofilm bacteria to antibiotics is due to the presence of large amounts of exopolysaccharides, the expression of biofilm-specific resistance genes, and suitable conditions for growing slowly (Rezaei et al., 2013). Methicillin-resistant Staphylococcus aureus is an important nosocomial pathogen that shows resistance to many antibiotics and has emerged as a serious threat to public health worldwide. On the other hand, biofilm formation ability makes MRSA exhibit stronger resistance to antibiotic agents, thus increasing difficulties in eradication of the pathogen from the host. In the current study, we found that the ethanol extract of $S$. officinalis inhibited biofilm formation ability of a MRSA strain. This indicates that, with the combined use of antibiotics and this medicine, the dose of antibiotic used in the treatment can be minimized. Our study provides new clues to the effective therapy for chronic infections caused by MRSA.

Sanguisorba officinalis is one kind of common Chinese medicine. Previous studies on S. officinalis have seldom reported its inhibitory effect on MRSA. In the present study, we found that $S$. officinalis remarkably inhibited the growth of MRSA at a high concentration $(>7.5 \mathrm{mg} / \mathrm{mL})$. Interestingly, we found that at a low concentration $(<2.5 \mathrm{mg} / \mathrm{mL})$, although $S$. officinalis only slightly inhibited the growth of the bacteria, the inhibitory effect of $S$. officinalis on biofilm formation in the MRSA strain was obvious. For example, the growth curve of the experimental group was slightly changed with the addition of $S$. officinalis at a concentration of $2.5 \mathrm{mg} / \mathrm{mL}$ compared with the control group without the addition of $S$. officinalis L.; however, at the same concentration, the MRSA strain almost lost the ability of biofilm formation.

Furthermore, based on detailed investigation into this interesting phenomenon, we found that the addition of S. officinalis at a low concentration can increase
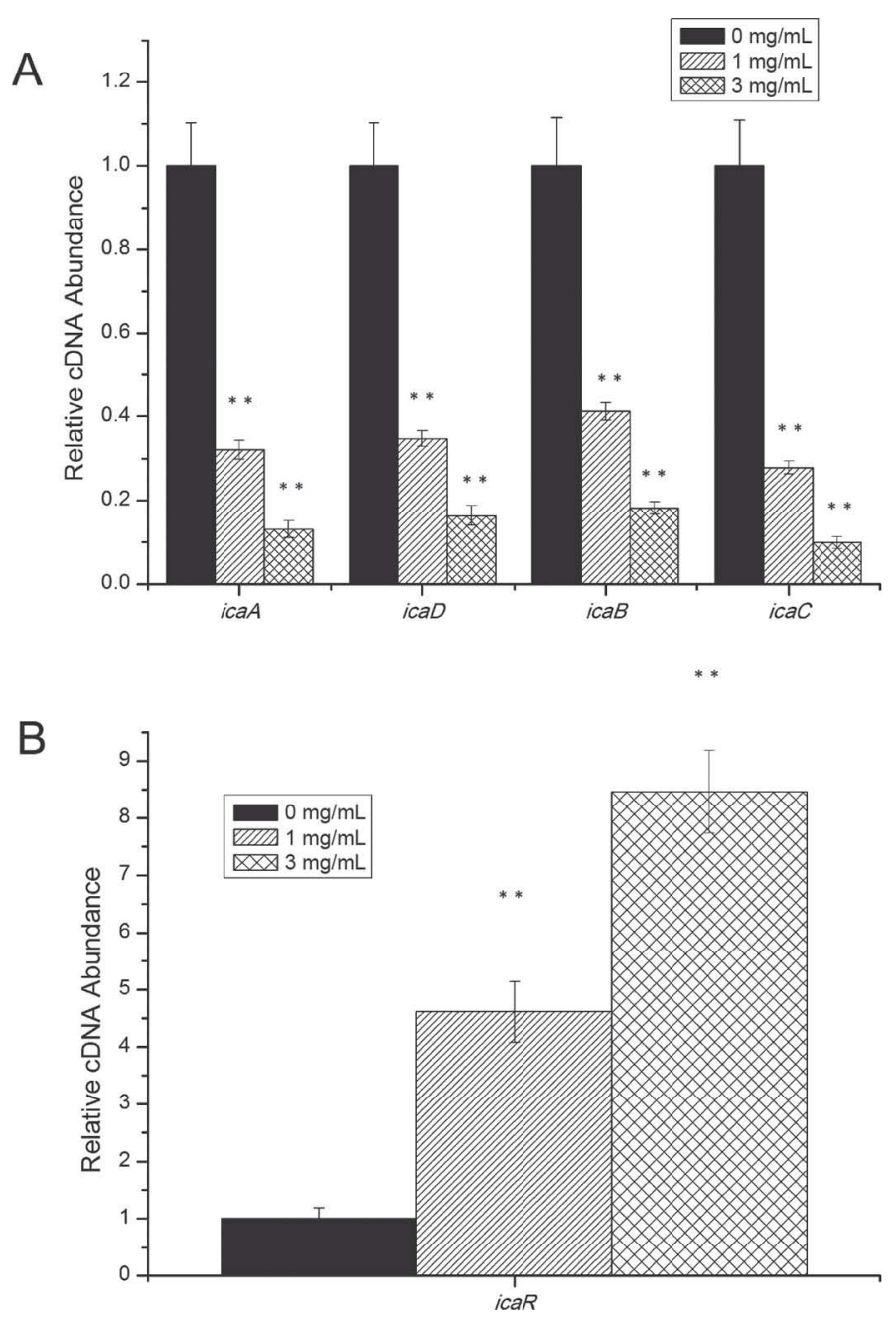

Figure 3. Comparative measurement of transcription of the biofilm-associated genes and their regulators. Relative transcript levels of icaA, icaB, icaC, and $i c a D$ and their regulatory gene icaR were determined by real-time reverse transcription PCR in methicillin-resistant Staphylococcus aureus (MRSA) strain SA3 cultured with or without ethanol extract of ethanol extract of Sanguisorba officinalis. Error bars indicate standard deviations. The results represent a mean of 3 independent experiments. Double asterisks $(* *)$ represent means significantly different from control group with no ethanol extract of $S$. officinalis $(P<0.01)$. 
the transcript level of $i c a R$, thus leading to the strong inhibitory effect on the transcription of the icaADBC operon, which encodes the polysaccharide intercellular adhesin synthetases. These data provide an explanation of how S. officinalis strongly inhibits the biofilm formation of MRSA only at low concentrations.

\section{ACKNOWLEDGMENTS}

This work was supported by the Project of Anhui Province Key Discipline of Biology (Anhui, China; 2014TSTD003; 2014SKQJ001) and the Talent Project of Anhui Agricultural University (YJ2013-6).

\section{REFERENCES}

Ando, E., K. Monden, R. Mitsuhata, R. Kariyama, and H. Kumon. 2004. Biofilm formation among methicillin-resistant Staphylococcus aureus isolates from patients with urinary tract infection. Acta Med. Okayama 58:207-214.

Atshan, S. S., M. N. Shamsudin, L. T. Lung, Z. Sekawi, E. GhaznaviRad, and C. P. Pei. 2012. Comparative characterisation of genotypically different clones of MRSA in the production of biofilms. J. Biomed. Biotechnol. 2012:417247.

Barrett, F. F., R. F. McGehee Jr., and M. Finland. 1968. Methicillin-resistant Staphylococcus aureus at Boston City Hospital. Bacteriologic and epidemiologic observations. N. Engl. J. Med. 279:441-448.

Bastow, K. F., I. D. Bori, Y. Fukushima, Y. Kashiwada, T. Tanaka, G. Nonaka, I. Nishioka, and K. H. Lee. 1993. Inhibition of DNA topoisomerases by sanguiin H-6, a cytotoxic dimeric ellagitannin from Sanguisorba officinalis. Planta Med. 59:240-245.

Cai, Z., W. Li, H. Wang, W. Yan, Y. Zhou, G. Wang, J. Cui, and F. Wang. 2012. Anti-tumor and immunomodulating activities of a polysaccharide from the root of Sanguisorba officinalis L. Int. J. Biol. Macromol. 51:484-488.

Chen, Y. W., P. Zhao, R. Borup, and E. P. Hoffman. 2000. Expression profiling in the muscular dystrophies: identification of novel aspects of molecular pathophysiology. J. Cell Biol. 151:1321-1336.

Fedtke, I., F. Gotz, and A. Peschel. 2004. Bacterial evasion of innate host defenses-the Staphylococcus aureus lesson. Int. J. Med. Microbiol. 294:189-194.

Gowrishankar, S., N. Duncun Mosioma, and S. Karutha Pandian. 2012. Coral-Associated bacteria as a promising antibiofilm agent against methicillin-resistant and -susceptible Staphylococcus aureus biofilms. Evid. Based Complement. Alternat. Med. 2012:862374.

Greenberg, D. P., A. S. Bayer, A. L. Cheung, and J. I. Ward. 1989. Protective efficacy of protein A-specific antibody against bacteremic infection due to Staphylococcus aureus in an infant rat model. Infect. Immun. 57:1113-1118.

Hussain, M., A. Haggar, C. Heilmann, G. Peters, J. I. Flock, and M. Herrmann. 2002. Insertional inactivation of Eap in Staphylococcus aureus strain Newman confers reduced staphylococcal binding to fibroblasts. Infect. Immun. 70:2933-2940.

Joh, D., E. R. Wann, B. Kreikemeyer, P. Speziale, and M. Hook. 1999. Role of fibronectin-binding MSCRAMMs in bacterial adherence and entry into mammalian cells. Matrix Biol. 18:211-223.

Liu, X., Y. Cui, Q. Yu, and B. Yu. 2005. Triterpenoids from Sanguisorba officinalis. Phytochemistry 66:1671-1679.

Mack, D., H. Rohde, S. Dobinsky, J. Riedewald, M. Nedelmann, J. K. Knobloch, H. A. Elsner, and H. H. Feucht. 2000. Identification of three essential regulatory gene loci governing expression of Staphylococcus epidermidis polysaccharide intercellular adhesin and biofilm formation. Infect. Immun. 68:3799-3807.

Matsuhashi, M., M. D. Song, F. Ishino, M. Wachi, M. Doi, M. Inoue, K. Ubukata, N. Yamashita, and M. Konno. 1986. Molecular clon- ing of the gene of a penicillin-binding protein supposed to cause high resistance to beta-lactam antibiotics in Staphylococcus aureus. J. Bacteriol. 167:975-980.

Nguyen, T. T., S. O. Cho, J. Y. Ban, J. Y. Kim, H. S. Ju, S. B. Koh, K. S. Song, and Y. H. Seong. 2008. Neuroprotective effect of Sanguisorbae radix against oxidative stress-induced brain damage: In vitro and in vivo. Biol. Pharm. Bull. 31:2028-2035.

O'Gara, J. P. 2007. ica and beyond: Biofilm mechanisms and regulation in Staphylococcus epidermidis and Staphylococcus aureus. FEMS Microbiol. Lett. 270:179-188.

Park, P. W., J. Rosenbloom, W. R. Abrams, and R. P. Mecham. 1996. Molecular cloning and expression of the gene for elastin-binding protein (ebpS) in Staphylococcus aureus. J. Biol. Chem. 271:1580315809.

Patti, J. M., B. L. Allen, M. J. McGavin, and M. Hook. 1994. MSCRAMM-mediated adherence of microorganisms to host tissues. Annu. Rev. Microbiol. 48:585-617.

Pozzi, C., E. M. Waters, J. K. Rudkin, C. R. Schaeffer, A. J. Lohan, P. Tong, B. J. Loftus, G. B. Pier, P. D. Fey, R. C. Massey, and J. P. O'Gara. 2012. Methicillin resistance alters the biofilm phenotype and attenuates virulence in Staphylococcus aureus device-associated infections. PLoS Pathog. 8:e1002626.

Rezaei, M., R. Moniri, S. G. A. Mousavi, and M. Jabari Shiade. 2013. Prevalence of biofilm formation among methicillin resistance Staphylococcus aureus isolated from nasal carriers. Jundishapur J. Microbiol. 6:e9601.

Sadovskaya, I., E. Vinogradov, S. Flahaut, G. Kogan, and S. Jabbouri. 2005. Extracellular carbohydrate-containing polymers of a model biofilm-producing strain, Staphylococcus epidermidis RP62A. Infect. Immun. 73:3007-3017.

Secor, P. R., G. A. James, P. Fleckman, J. E. Olerud, K. McInnerney, and P. S. Stewart. 2011. Staphylococcus aureus Biofilm and Planktonic cultures differentially impact gene expression, mapk phosphorylation, and cytokine production in human keratinocytes. BMC Microbiol. 11:143.

Shanks, R. M., N. P. Donegan, M. L. Graber, S. E. Buckingham, M. E. Zegans, A. L. Cheung, and G. A. O'Toole. 2005. Heparin stimulates Staphylococcus aureus biofilm formation. Infect. Immun. 73:4596-4606.

Stewart, P. S. 2002. Mechanisms of antibiotic resistance in bacterial biofilms. International journal of medical microbiology. Int. J. Med. Microbiol. 292:107-113.

Ubukata, K., N. Yamashita, and M. Konno. 1985. Occurrence of a beta-lactam-inducible penicillin-binding protein in methicillin-resistant staphylococci. Antimicrob. Agents Chemother. 27:851-857.

Yokozawa, T., C. P. Chen, T. Tanaka, and K. Kitani. 2000. A study on the nitric oxide production-suppressing activity of sanguisorbae radix components. Biol. Pharm. Bull. 23:717-722.

Yokozawa, T., C. P. Chen, T. Tanaka, and K. Kitani. 2002. Effects of sanguiin H-6, a component of Sanguisorbae Radix, on lipopolysaccharide-stimulated nitric oxide production. Biochem. Pharmacol. 63:853-858.

Yu, D., L. Zhao, T. Xue, and B. Sun. 2012. Staphylococcus aureus autoinducer-2 quorum sensing decreases biofilm formation in an icaR-dependent manner. BMC Microbiol. 12:288.

Yu, T., Y. J. Lee, H. M. Yang, S. Han, J. H. Kim, Y. Lee, C. Kim, M. H. Han, M.-Y. Kim, J. Lee, and J. Y. Cho. 2011. Inhibitory effect of Sanguisorba officinalis ethanol extract on NO and PGE2 production is mediated by suppression of NF- $\kappa \mathrm{B}$ and AP-1 activation signaling cascade. J. Ethnopharmacol. 134:11-17.

Zhang, L., S. R. Koyyalamudi, S. C. Jeong, N. Reddy, P. T. Smith, R. Ananthan, and T. Longvah. 2012. Antioxidant and immunomodulatory activities of polysaccharides from the roots of Sanguisorba officinalis. Int. J. Biol. Macromol. 51:1057-1062.

Ziebuhr, W., C. Heilmann, F. Gotz, P. Meyer, K. Wilms, E. Straube, and J. Hacker. 1997. Detection of the intercellular adhesion gene cluster (ica) and phase variation in Staphylococcus epidermidis blood culture strains and mucosal isolates. Infect. Immun. 65:890896 